07

\title{
Инжекция эмиссионных электронов в мультизеренной наноструктуре полупроводников
}

\author{
() Н.Д. Жуков ${ }^{1}$, А.А. Хазанов ${ }^{1}$, Я.Е. Переверзев ${ }^{2}$ \\ ${ }^{1}$ ООО „Реф-Свет“, Саратов \\ ${ }^{2}$ Саратовский государственный университет им. Н.Г. Чернышевского \\ E-mail: ndzhukov@rambler.ru
}

Поступило в Редакцию 27 декабря 2016 г.

Методами аппроксимации экспериментальных ВАХ исследован механизм инжекции эмиссионных электронов и сделан вывод о том, что инжекция в монокристаллическую и мультизеренную полупроводниковые структуры может быть описана одной физической моделью, состоящей в туннельном преодолении электронами поверхностного барьера и диффузионно-дрейфовом транспорте неравновесных электронов в полупроводнике. Определяющей закономерностью ВАХ является степенная зависимость с показателями степени от 2 до 4. Анализ ВАХ позволяет оценить произведение величин подвижности и диффузионной длины неравновесных электронов. Результаты могут быть использованы в исследованиях и при разработках мультизеренных структур для газовых и оптических сенсоров, приемников и излучателей инфракрасного и терагерцевого диапазонов.

DOI: 10.21883/PJTF.2017.12.44703.16644

Одним из направлений наноиндустрии является создание и использование структур на основе субмикронных полупроводниковых частиц [1-4]. В связи с развитием этих направлений важным является изучение процессов межзеренных и межслойных эмиссии и инжекции электронов [5,6]. Интерес при этом представляет получение и исследование инжекции эмиссионных электронов. Такие физические процессы являются определяющими для свойств мультизеренных и мультикристаллических структур, которые могут быть использованы в газовых и оптических сенсорах, источниках и приемниках дальнеинфракрасных излучений. 


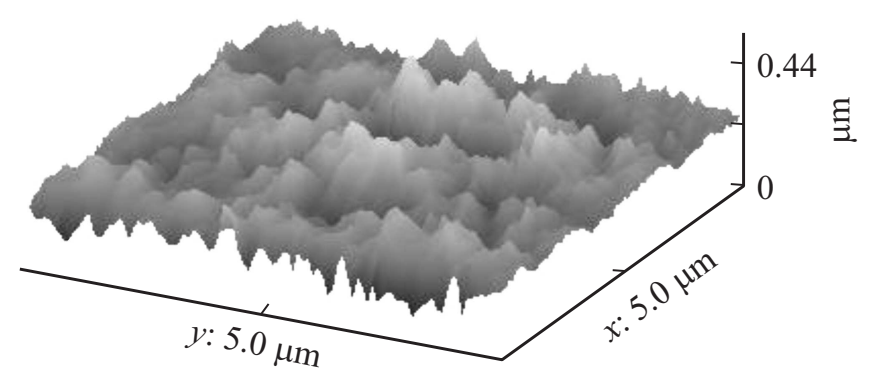

Рис. 1. Тунельно-токовая 3D-топограмма полированной поверхности полупроводников $\mathrm{GaAs}$ и $\mathrm{Si}$.

В данной работе, в продолжение предыдущих публикаций [7-9], исследовано явление инжекции эмиссионных электронов в субмикронные зерна наиболее применяемых полупроводников - кремния $\mathrm{Si}$, арсенида галлия GaAs, арсенида InAs и антимонида InSb индия. Изучены процессы транспорта электронов в двух вариантах: в эмиссионнотуннельном локальном контакте с нанозазором микрозонд-микрозерно поверхности полупроводника (вариант СТМ) и вакуумной триодной структуре термокатод-сетка-анод с мультизеренным анодным слоем (вариант ВТС). Методики подготовки образцов и измерений характеристик описаны в работах [7,8]. Использованы монокристаллические подложки электронной проводимости с финишной обработкой алмазным порошком АМП1 для InAs, InSb и GaAs и химико-механической полировкой (ХМП) для Si и GaAs. Максимально „тонкую“ (до $100 \mathrm{~nm}$ ) мультизеренную структуру поверхности имели подложки с ХМП (рис. 1). Поверхность подложек с АМП-шлифовкой имела структуру зерен с размерами от 0.2 до $2 \mu \mathrm{m}$.

Субмикронные порошки для варианта ВТС изготавливались измельчением монокристаллических подложек в шаровой мельнице Pulverisette-7. С учетом оценок предела прочности субмикронных частиц с использованием модели пластической деформации и соотношения Холла-Петча [10] полагалось, что полученные частицы с размерами на минимальном пределе не имели существенно нарушенной структуры по отношению к исходным монокристаллам. Методом седиментации проводился отбор субмикронных частиц с размерами на мини-

Письма в ЖТФ, 2017, том 43, вып. 12 
мальном пределе. Покрытия из нанопорошков формировались методом катафореза из суспензии в изопропиловом спирте. Толщина слоя покрытия составляла порядка $5 \mu \mathrm{m}$. Средние размеры частиц в зависимости от типа полупроводника были для $\mathrm{InSb}-0.37 \mu \mathrm{m}$, для остальных $0.22-0.23 \mu \mathrm{m}$, отклонения размеров в пределах $0.1-0.2 \mu \mathrm{m}$, удельная поверхность $-15-25 \mu \mathrm{m}^{-1}$.

В использованной эмиссионно-инжекционной структуре ток включает несколько последовательных процессов: эмиссия электронов из катода (,эмиссия“), пролет межэлектродного пространства катод-анод $($ „пролет“), преодоление потенциального барьера поверхности образца („инжекция“), диффузионно-дрейфовый транспорт в полупроводнике („диффузия“), электрический ток через толщу полупроводника и область контактирования к нему электрода („электроток“). Каждый из этих процессов может лимитировать ток и тем самым быть отображен на определенном участке вольт-амперной характеристики (BAX) и детально исследован.

Вычисления сопротивления толщи полупроводника, оценки свойств контактов к используемым полупроводникам (по литературным и собственным данным) показали, что для всех экспериментов процесс ,электроток“ не являлся лимитирующим. Благодаря большой подвижности электронов в используемых в работе полупроводниках, процесс „диффузия“ может быть лимитирующим в случае возникновения ограничения тока пространственным зарядом, что наблюдалось при относительно больших значениях напряжения, при которых процессы „эмиссия“ и „инжекция“ не лимитировали ток [7].

Из двух основных для данного исследования лимитирующих ток процессов — „эмиссия““ и ,инжекция“ - первый проявится на ВАХ при относительно больших величинах напряженности поля $E$, когда „инжекция“ не будет лимитирующей. Исследования на туннельном микроскопе по методике, описанной в работах [7,9], позволили установить области ВАХ и параметры процесса эмиссии электронов из зонда. На большом числе образцов при разных значениях величин зазора зонд-образец получены статистические данные по величинам наклона прямых для ВАХ в координатах Фаулера-Нордгейма $\left(\ln I / V^{2}-1 / V\right)-$ $\sim(1-2) \mathrm{V} / \mathrm{nm}$ и отсечки на оси логарифм-ток $\operatorname{Ln} I_{0} \sim(13-15)$. По этим данным и формуле Фаулера-Нордгейма [11] $I \sim$ $\sim 1.55 \cdot 10^{-8} k^{2} E^{2} \varphi^{-1} S \cdot \exp \left[-6.85 \cdot 10^{7} \varphi^{3 / 2} k^{-1} E^{-1}\right] \mathrm{A}$, проведены оценки площади $S$ круга стекания-эмиссии электронов с поверхности

Письма в ЖТФ, 2017, том 43, вып. 12 
зонда и форм-фактора $k$ усиления поля на нем: $k \sim(35-70) \mathrm{nm}^{-1}, S \sim$ $\sim(3-5) \mathrm{nm}^{2}$. Вычисленные при этом значения площади круга инжекции $(\sim k S)$ на поверхности полупроводника $S_{0} \sim\left(10^{2}-10^{3}\right) \mathrm{nm}^{2}$.

Инжекция электронов в полупроводник происходит путем преодоления потенциального барьера, образованного наличием на его поверхности супертонкой пленки окисла (с некими примесями) и возможного приповерхностного слоя обеднения или обогащения. При этом возможны несколько механизмов преодоления барьера и соответствующие им формулы ВАX [12-14]: надбарьерная эмиссионная (тепловая) инжекция $-I \sim \exp (q V / k T)$; туннелирование сквозь барьер $I \sim \exp (A V)$; надбарьерная эмиссия электронов по механизму Шоттки $I \sim \exp \left(\beta E^{1 / 2}\right)$, где $\beta=(4 \pi \varepsilon)^{-1 / 2} q / k T \sim 12$.

BAX всех исследованных образцов имели явно выраженные участки - при относительно больших, средних и малых величинах напряжения. В области относительно больших величин напряжения выполнялись соотношения полевой эмиссии для варианта СТМ и закона трех вторых (Ленгмюра) для варианта ВТС. Кроме того, для варианта СТМ могли наблюдаться участки $\mathrm{BAX}$, которые хорошо аппроксимировались формулой $I \sim \exp \left(\beta E^{1 / 2}\right)$ при значениях $\beta \sim(11-13)$, хорошо соответствующих теории.

На рис. $2, a, b$ приведены ВАХ образцов в координатах $\operatorname{Ln} I \sim V$ в области относительно малых значений тока и напряжения. BAX аппроксимируются формулой туннелирования сквозь барьер $I \sim \exp (A V)$. При этом BAX интерпретируются механизмом прямого туннелирования электронов сквозь супертонкий барьер $[12,13]$. Правильность такой аппроксимации подтверждается также и слабой температурной зависимостью показателя экспоненты - угла наклона прямых на рис. $2, b$. При этом теория дает выражение для параметра $A \sim d^{*}\left[\left(4 m^{*} q\right)\left(9 h^{2} k T\right)^{-1}\right]^{1 / 2}[13]$, где $m^{*}$ и $q-$ эффективная масса и заряд электрона, $h-$ постоянная Планка, $k$ и $T-$ постоянная Больцмана и абсолютная температура, $d^{*}$ - толщина барьерного слоя. Вычисления приводят к выражению для значений $A-A \sim 2.5 d^{*}$. По ВАХ (рис. $2, a$ ) найдены среднестатистические значения параметра $A$ и соответствующие им величины $d^{*}(\mathrm{~nm})$ для варианта СТМ: $\mathrm{Si}-\sim 7\left(d^{*} \sim 2.8\right)$; GaAs $-\sim 6\left(d^{*} \sim 2.4\right)$; InAs $-\sim 11\left(d^{*} \sim 4.1\right)$; InSb $-\sim 6\left(d^{*} \sim 2.4\right)$. Для варианта ВТС величины параметров были заметно меньше $-\sim 0.7$ (рис. $2, b)$. Это, а также некоторое уменьшение $A$ с ростом величины 

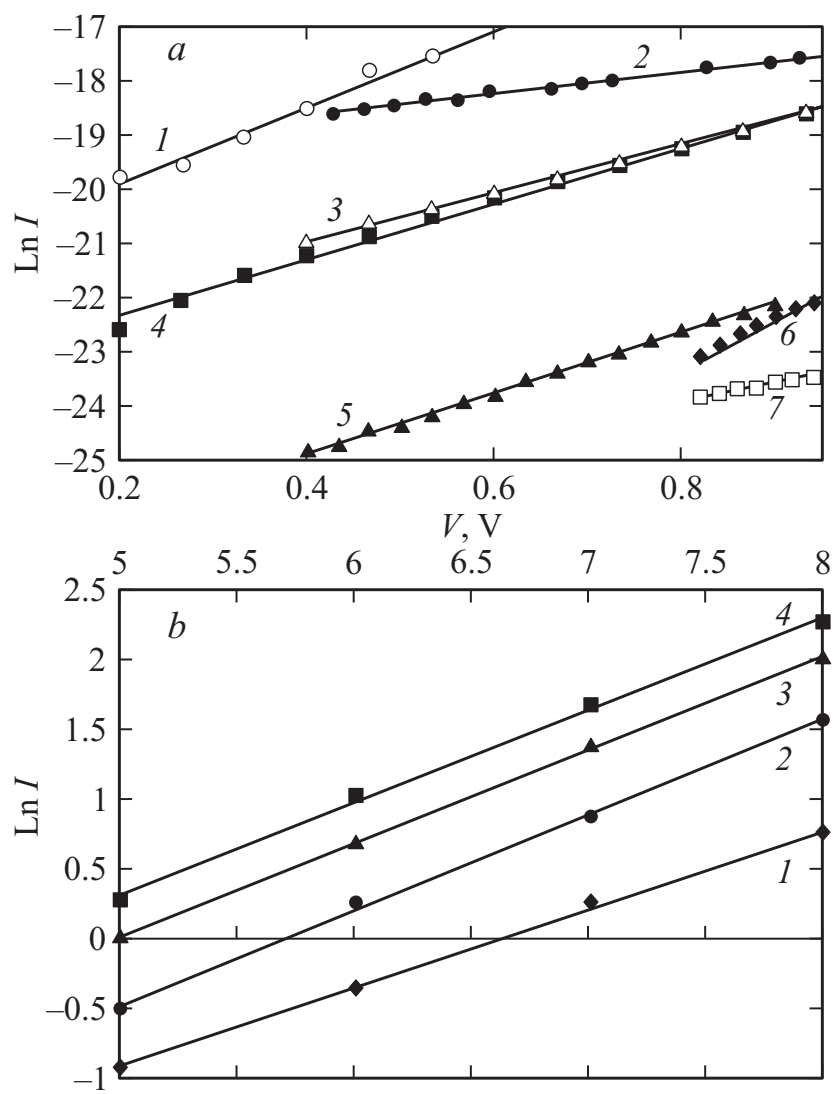

Рис. 2. Типичные BAX в полулогарифмических и логарифмических координатах. $a, c-$ вариант СТМ, $b, d-$ вариант ВТС $\left(d=1 \mathrm{~mm}, S=1 \mathrm{~cm}^{2}\right.$, $U_{g}-$ напряжение сетка-катод). Единицы параметров: $V$ и $V_{g}-[\mathrm{V}] ; g-[\mathrm{nm}]$; $T-[\mathrm{K}] . a: 1-\mathrm{Si}, d=0 ; 2-\mathrm{Si}, d=3 ; 3-\mathrm{InAs}, d=0 ; 4-\mathrm{InSb}, d=0 ; 5-$ InAs, $d=5 ; 6-\mathrm{GaAs}, d=0 ; 7-\mathrm{InSb}, d=3 . b: 1-U_{g}=0, T=293 ; 2-$ $U_{g}=20, T=293 ; 3-U_{g}=20, T=313 ; 4-U g=20, T=353 . c: 1-$ GaAs, $d=5 ; 2-\mathrm{GaAs}, d=2 ; 3-\mathrm{InSb}, d=3 ; 4-\mathrm{InSb}, d=6 ; 5-\mathrm{InSb}, d=9 . d$ : $1-U g=0 ; 2-U_{g}=20 ; 3-U_{g}=100$.

зазора для варианта СТМ, объясняются перераспределением падения напряжения в пользу межэлектродного зазора.

Письма в ЖТФ, 2017, том 43, вып. 12 

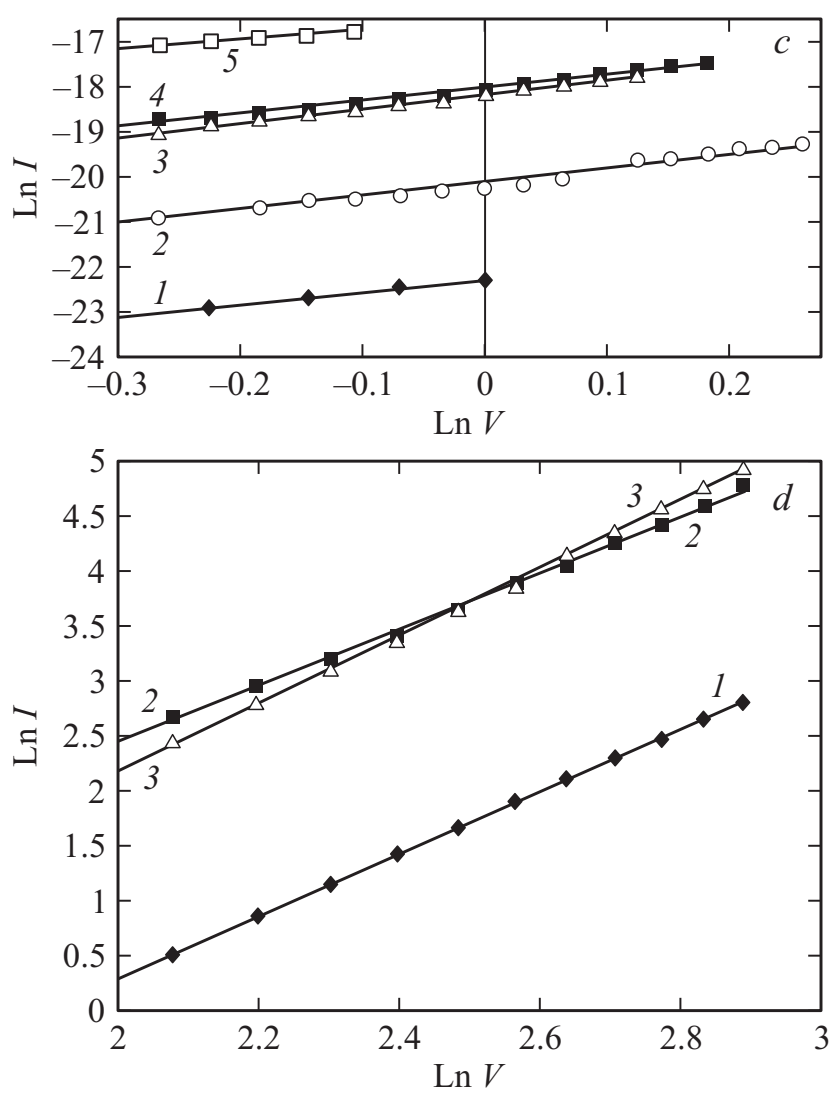

Рис. 2 (продолжение).

Транспорт инжектированных в приповерхностную зону полупроводника электронов выражается формулой для плотности тока $J_{S}$, которая получается решением уравнения непрерывности [14]: $J_{S}=q \mu[n E+(k T / q) d n / d x]$, где $\mu$ и $n-$ соответственно подвижность и концентрация инжектированных электронов, $E$ - поле в полупроводнике. Инжектированные в полупроводник электроны создают в нем неравновесный заряд, который „рассасывается“ путем дрейфа и диффузии неравновесных электронов и их рекомбинации с дырками. При этом 
для данного случая полупроводников темп рекомбинации и градиенты концентрации пропорциональны величине концентрации неравновесных носителей [14]: $d n / d x=-n / L$, где $L$-размерная постоянная — диффузионная длина для неравновесных (инжектированных) электронов [14]. Интегрирование приведет к распределению концентрации неравновесных электронов $-n=n_{0} \exp (-x / L)$, где $n_{0}-$ концентрация неравновесных электронов на границе-поверхности полупроводника. Решение уравнения Пуассона $d E / d x=q n / \varepsilon$ в этом случае дает распределение напряженности поля: $E=\left(q L n_{0} / \varepsilon\right) \exp (-x / L)$. Подставляя выражения для $n, d n / d x, E$ в формулу для $J_{S}$, получится для координаты $x=0$ выражение для плотности тока: $J_{S}=q^{2} \mu n_{0}^{2} L \varepsilon^{-1}\left[1+k T \varepsilon\left(q^{2} L^{2} n_{0}\right)^{-1}\right]$. Второе слагаемое по оценке меньше единицы при $n_{0}>10^{11} \mathrm{~cm}^{-3}$. Проверяя и подтверждая во всех случаях выполнение этого условия, вторым слагаемым пренебрегалось. Тогда ВАХ выразится формулой $J_{S}=q^{2} \mu L \varepsilon^{-1} n_{0}^{2}$, в которой зависимость от внешнего напряжения определяется величиной $n_{0}^{2}$. Значения $n_{0}$ находятся из выражения для потока электронов между анодом и катодом для варианта ВТС и между зондом и образцом для варианта СТМ в предположении, что концентрация электронов в зазоре постоянна $-J_{0}=q v n_{0}$, где $v-$ скорость электронов между электродами $-v^{2}=2 q m^{-1} V^{*}, V^{*}-$ разность потенциалов между электродами. Вычисления дают выражение для BAX: $J_{S}=0.5 q^{-1} m \mu L \varepsilon^{-1} V^{*-1} J_{0}^{2}$.

Ток в вакууме между термокатодом и анодом (вариант ВТС) соответствует формуле Ленгмюра [11] $J_{0} \sim 0.6 q^{1 / 2} \varepsilon_{0} m^{-1 / 2} d^{-2} V^{3 / 2}$, где $d-$ расстояние между электродами. Для варианта экстремально малого расстояния между электродами - нанозазора $d$ в варианте СТМ - можно использовать модель прыжковой проводимости и из формул Мотта и Ламперта $[14,15]$ найти $J_{0}: J_{0} \sim 0.1 \varepsilon_{0} v d^{-2} V^{2}$. В итоге вычисления дают следующее выражение для тока $I$ :

$$
I=c \alpha S V^{g},
$$

где $\alpha=\mu \varepsilon_{0}\left(\varepsilon / \varepsilon_{0}\right)^{-1} L d^{-4} V^{*-1}$. Для варианта ВТС $-c \sim 0.2$ и $g \sim 3$; для варианта СТМ $-c \sim 7 \cdot 10^{-3} V^{*-1}$ и $g \sim 4$.

Для варианта СТМ величины межэлектродного зазора зонд-образец $d$ много меньше $L$ [16], что позволяет принять величину $L$ в формуле (1) как $d$, а $V^{*} \sim V-$ почти полное падение напряжения на образце. Тогда в формуле (1) $g \sim 3$, а выражение для $\alpha$

Письма в ЖТФ, 2017, том 43, вып. 12 
примет вид $\alpha=\mu \varepsilon_{0}\left(\varepsilon / \varepsilon_{0}\right)^{-1} L^{-3}$. В этом случае обработка массива экспериментальных результатов для ВАХ типа рис. 2, с позволила получить: $g \sim 3, \ln (c \alpha S) \sim-(18-19), \mu S L^{-3} V^{*-1} \sim\left(10^{4}-10^{5}\right)$. Используя значения $\mu \sim 10 \mathrm{~m}^{2} \cdot \mathrm{V}^{-1} \cdot \mathrm{s}^{-1}, L \sim 10^{-6} \mathrm{~m}$ [16] и приняв $V^{*} \sim 0.1 \mathrm{~V}$, для величины площади $S$ токопротекания в образце получится $S_{0} \sim\left(10^{2}-10^{3}\right) \mathrm{nm}^{2}$, что соответствует данным, полученным другим способом и приведенным выше.

Для варианта ВТС обработка ВАХ типа рис. 2, $d$ образцов полупроводников $\mathrm{A}_{3} \mathrm{~B}_{5} \mathrm{c}$ аппроксимацией формулой (1) позволила получить данные для величин $g$ и $\mu L: g \sim 3, \mu L \sim 10^{-4}$. В данном случае, принимая за величину $L$ размер толщины мультизеренного слоя $\sim 10^{-5} \mathrm{~m}$, получено для величин подвижности электронов в мультизеренной структуре $\mu \sim 10 \mathrm{~m}^{2} \cdot \mathrm{V}^{-1} \cdot \mathrm{s}^{-1}$. Получение таких высоких значений подвижности, принципиально свойственных полупроводникам $\mathrm{A}_{3} \mathrm{~B}_{5}$ с легкими электронами [16], кроме того, может означать, что субмикронные частицы мультизеренной слоистой структуры имеют высокое структурное совершенство.

Таким образом, полученные результаты позволяют сделать вывод о том, что инжекция эмиссионных электронов в монокристаллическую и мультизеренную полупроводниковые структуры происходит по единому механизму. Физические модели инжекции заключаются в основном в туннельном преодолении электронами поверхностного барьера и диффузионно-дрейфовом транспорте неравновесных электронов в полупроводнике. При этом основным типом ВАХ является степенная зависимость с показателями степени от 2 до 4. Анализ ВАХ позволяет оценить произведение величин подвижности и диффузионной длины неравновесных электронов в полупроводниковой структуре.

Исследование выполнено при финансовой поддержке РФФИ в рамках научного проекта 16-07-00093-а.

\section{Список литературы}

[1] Третьяков Ю.Д. // Нанотехнологии. Экология. Производство. 2011. № 1(8). C. 98.

[2] Глухова О.Е., Гороховский А.В., Жуков Н.Д. и др. Основы наноиндустрии. Саратов: Изд-во Сарат. ун-та, 2009. 384 с. 
[3] Пещерова С.М., Непомнящих А.М., Павлов Л.А. и др. // ФТП. 2014. Т. 48. B. 4. C. 492.

[4] Бобков А.А., Максимов А.И., Мошников В.А. и др. // ФТП. 2015. Т. 49. В. 10. C. 1402.

[5] Витухновский А.Г., Ващенко А.А., Бычковский Д.Н. и др. // ФТП. 2013. T. 47. B. 12. C. 1591.

[6] Achermann M., Petruska M.A., Kos S. et al. // Nature. 2004. V. 429. P. 642.

[7] Жуков Н.Д., Глуховской Е.Г., Хазанов А.А. // ФТП. 2016. Т. 50. В. 6. С. 772.

[8] Жуков Н.Д., Мосиям Д.С., Хазанов А.А. и др. // Прикладная физика. 2015. № 3. C. 93.

[9] Глуховской Е.Г., Жуков Н.Д. // Письма в ЖТФ. 2015. Т. 41. В. 14. С. 47.

[10] Глезер А.М., Столяров В.Л., Томчук А.А. и др. // Письма в ЖТФ. 2016. Т. 42. B. 1. C. 103.

[11] Егоров Н.В., Шешин Е.П. Электронная эмиссия. М.: Интеллект, 2011.

[12] Гольдман Е.И., Гуляев Ю.В., Ждан А.Г. и др. // ФТП. 2010. Т. 44. В. 8. C. 1050.

[13] Милнс А., Фойхт Д. Гетеропереходы и переходы металл-полупроводник. М: Мир, 1975.

[14] Ламперт М., Марк П. Инжекционные токи в твердых телах. М.: Мир, 1973.

[15] Мурсататов Ш.А., Лейдерман А.Ю., Айтбаев Б.У. и др. // ФТТ. 2009. Т. 51. B. 10. C. 1917.

[16] Маделунг О. Физика полупроводниковых соединений элементов III и V групп. М.: Мир, 1967.

2 Письма в ЖТФ, 2017, том 43, вып. 12 